\title{
Impact of changes in community psychiatric service provision on mental health presentations to the emergency department
}

\author{
Caoimhe McLoughlin ${ }^{1} \cdot$ Ahad Abdalla $^{2} \cdot$ Siobhan MacHale ${ }^{1} \cdot$ Helen Barry $^{1}$ \\ Received: 13 August 2020 / Accepted: 18 November 2020 / Published online: 21 November 2020 \\ (C) Royal Academy of Medicine in Ireland 2020
}

\begin{abstract}
Background The reconfiguration of many Irish stand-alone psychiatric units has led to many patients in acute mental health need now being assessed in emergency departments (EDs). This has implications for ED resources and raises questions about appropriate assessment location for this group.

Aims This report aims to examine the impact of removal of a direct community access point for patients in acute mental health need on ED presentations in a Dublin hospital.

Methods We examined data on ED referrals to psychiatry over 5 years: 12 months before the service change, and four subsequent 12-month periods. We compared numbers referred, mode of referral, average ED length of stay, proportion with no physical issue requiring psychiatric assessment only, and numbers who did not wait for psychiatry assessment.

Results In the year directly after the service change, referrals to psychiatry from ED increased by $200 \%$, remaining at this level for the subsequent 3 years. Of these, $32.5 \%$ were referred by a GP-more than a threefold increase in numbers from the previous year, with both numbers remaining similarly elevated over subsequent years. In the year after the service change, $52.1 \%$ of total ED to psychiatry presented solely for mental health reasons - nearly a fourfold increase in cases from the previous year, and remained high.

Conclusions Removing a direct community access point for this group resulted in a substantial increase in ED presentations, many of which did not have physical needs. This study has implications for future policy to address the needs of this group, especially in light of the pandemic.
\end{abstract}

Keywords Emergency department $\cdot$ Liaison psychiatry $\cdot$ Mental health

\section{Introduction}

\section{Where should psychiatric emergencies be seen?}

There is no clear international consensus on where patients with acute mental health needs should be assessed. International guidelines regarding the optimal setting for emergency psychiatric assessment vary, but common emphasis is placed on the provision of a thoughtfully designed, designated safe space for

Caoimhe McLoughlin

cmcloug6@tcd.ie

1 Department of Liaison Psychiatry, Beaumont Hospital, Beaumont Rd, Dublin 9, Ireland

2 Department of Medicine, University Limerick Hospital Group, Limerick, Ireland patients, with prompt assessment by skilled specialists [1-3]. In the Irish context, there are clear established frameworks for the treatment of patients who experience mental health difficulty in the emergency setting, which state that patients in mental health crisis, without physical need, should have direct access to their local community psychiatric teams over a 24 -h period, without recourse to the ED $[4,5]$. Furthermore, it has been recognised that when mental health services rely on ED to urgently assess patients as opposed to community services, higher rates of direct inpatient psychiatric admissions result, with obvious cost implications [6].

There are some who may argue that psychiatric patients are just like other patients who present to the ED and therefore should be processed through the standard ED care pathway. Zun [7] outlines the different and unique needs of psychiatric patients and the challenges posed by conventional ED management of mental health presentations. It is without question that the ED is the most appropriate place of referral for certain 
psychiatric emergencies such as cases of self-harm requiring emergency medical treatment. Furthermore, in cases of atypical psychosis or delirium, appropriate systemic work-up is crucial $[8,9]$. However, there are a proportion of patients who do not require the attention of an emergency medical physician, where a prompt biopsychosocial assessment from a trained mental health specialist will most appropriately meet their needs in the acute setting and prevent necessary treatment delays. Furthermore, it is important to optimise community emergency pathways as much as possible, as crowding in the ED is a worsening problem which impacts on patient safety and quality of care [10-12]. Previous studies have reported that the length of stay in ED and number of visits for patients undergoing psychiatric evaluation in the ED increased significantly after the closure of county inpatient psychiatric services [13], and it has been shown in some jurisdictions that such problems may be alleviated by alternative care pathways that integrate primary care, community and liaison-psychiatry services $[14,15]$.

\section{Context of study}

The closure of a "stand-alone" psychiatric unit in North County Dublin, and its relocation to a general hospital campus in May 2014 resulted in a redirection of local emergency psychiatry presentations directly to the general hospital ED, as distinct from the stand-alone off-site unit which had previously catered for direct assessments from the community. This move was not a closure of services for psychiatric patients, but instead provided emergency psychiatric assessment in a different location. While data on waiting times for patients in the stand-alone unit are not available, it is of note that patients when they were seen there, were assessed directly by a mental health specialist in an acute mental health assessment unit, and did not have to go through medical triage, nor did they undergo an additional assessment by an ED clinician nor wait in a busy ED environment.

\section{Aim}

This report aims to examine the impact of removal of a direct community access point for patients in acute mental health need on ED presentations in a Dublin tertiary referral centre.

\section{Methods}

\section{Setting}

Beaumont Hospital is a large tertiary referral hospital situated $5 \mathrm{~km}$ north of Dublin City, with a capacity of 820 beds. The offsite inpatient psychiatric unit prior to its closure directly accepted self-referrals and GP referrals for patients with acute mental health needs and served the North County Dublin community population of approximately 250,000 [16]. Prior to closing, the off-site unit assessed approximately $40-45$ patients per month (480-540 per year). In May 2014, this unit closed, and a new inpatient psychiatric unit was built on the site of the main Beaumont Hospital Campus, adjacent to the general hospital, resulting in a redirection of all mental health presentations through the ED. Regarding a shift in resources to accommodate these changes, there was an associated expansion of the on-call psychiatry services (comprising a psychiatry registrar and consultant) to include cover to the ED out of hours. Additionally, 7 months after the change, self-harm nurses were appointed coincident with the implementation of the National Clinical Programme (NCP) for Patients presenting to ED following Self- Harm [5]. This program is a joint initiative between the Department of Health and the College of Psychiatrists of Ireland, with a remit to provide for the assessment and management of patients who present to emergency departments following self-harm, and included the appointment of extra nursing staff in the ED [5]. It was introduced in Beaumont Hospital in December 2014 and includes 2.5 full-time equivalent clinical nurse specialist posts.

\section{Data collection and analysis}

We retrospectively examined data on referrals to psychiatry from Beaumont Hospital Emergency Department over 5 years: 12 months immediately before the service change (May 20132014) and the four subsequent 12-month periods, (May 2014 2015, May 2015-2016, May 2016-2017, May 2017-2018). All patients referred to the psychiatry service by the ED during the specified time periods were included. Patient data is recorded by ED and liaison psychiatry team members on the hospital's coding and electronic patient record (EPR) digital system. Selected data relevant to the time periods specified were obtained by the Beaumont Hospital Management Information Officer using Driver 7, a business intelligence data analysis and reporting tool, which was used to extract anonymised data from the psychiatry EPR clinical database prior to being evaluated by the authors. We compared caseload and referral trends over these five periods. The following was recorded by the authors and used as outcome measures: numbers of patients referred to psychiatry from ED, mode of referral to ED (self, GP, or other), average length of stay (LOS) in the ED, proportion with no physical issue identified at triage requiring mental health assessment only, and numbers who did not wait for psychiatric assessment. The project received approval from the Clinical Governance, Audit and Service Evaluation Committee (Registered Number CA448). This was a service evaluation project, and no personal individual patient data was collected to protect patient confidentiality. All analyses were carried out on IBM Statistical Package 
for Social Sciences ${ }^{\circledR}$ (SPSS) Version 26. A $p$ value $<0.05$ was used as the level of significance.

\section{Results}

\section{Numbers referred to psychiatry from ED}

In the year directly before the unit opened, 544 patients were referred to psychiatry by an ED clinician. In the year directly after the opening, numbers more than doubled, with 1118 patients referred to psychiatry. In the subsequent 3 years, the numbers remained at $200 \%$ of those seen prior to the move, with total numbers for each subsequent 12-month period at 1075, 1049, and 1062 respectively. See Table 1.

\section{Mode of referral to ED}

In the year directly before the unit opened, $77 \%(n=419)$ of patients self-presented to the ED. $15.8 \%(n=86)$ were referred by a GP and the remainder $7.1 \%(n=39)$ were classified as "referred from other" (including referred internally, from a voluntary agency, or the police department).

In the year directly after the unit opened, 664 (59.4\%) selfpresented to the ED, an increase of $58 \%$ in numbers from the previous year. A total of 363 (32.5\%) were referred by a GPthis was more than a threefold increase in numbers from the previous year and a proportional increase of $16.6 \%$.

Over the following 3 years, the number of self-referrals remained elevated at 690 (64.2\% of total), 667 (63.6\%), and $701(66 \%)$ respectively. GP referrals were also persistently elevated at 308 (28.7\%), 289 (27.6\%), and 301 (28.3\%). The change in referral trends was a statistically significant difference $(p<0.001)$ for both GPs and self-referral. See Table 1.

\section{Number with physical issue identified at triage}

In the year before the unit opened, 29.2\% $(n=159)$ had no physical issue identified at triage. In the following year, $52.1 \%$ $(n=582)$ had no physical issue identified at triage-nearly a fourfold increase in cases, and a proportional increase of $22.9 \%$. This was statistically significant $(p<0.001)$. Over the following 3 years the proportion presenting with no physical issue identified at triage remained similar, at $52.4 \%(n=$ $563), 52.1 \%(n=547)$, and $50.8 \%(n=539)$ respectively. See Table 1.

\section{Length of stay in ED}

In the year before the unit opened, the average LOS in the ED was $14.9 \mathrm{~h}$. In the year after the unit opened, this decreased to $11.1 \mathrm{~h}$. In the subsequent 3 years, the average LOS in ED stood at $11.6,10.1$, and $10.4 \mathrm{~h}$, respectively. This was statistically significant $(p<0.001)$ in pairwise comparison between the year before the unit opened and each subsequent year. These changes are likely to be due to the impact of the NCP for self-harm [5]. See Table 1.

\section{Numbers who did not wait for psychiatric assessment}

In the year prior to the unit opening, $2 \%(n=11)$ did not wait for psychiatric assessment. The following year after opening, this increased slightly to $2.3 \%(n=26)$. Over the following 3 years, the proportion who did not wait decreased slightly to $1.5 \%(n=16), 0.8 \%(n=8)$, and $1.6 \%(n=17)$. This did not reach statistical significance $(p=0.06)$. See Table 1 .

\section{Discussion}

Our results show that the service change resulted in a doubling of patients whose emergency mental health assessments were carried out in the ED setting - resulting in a substantially increased additional workload into the already overstretched ED environment. Though extra resources were allocated to the ED out of hours to assess patients with self-harm following the service relocation, lack of direct access to emergency psychiatric services resulted in much higher volume of patients

Table 1 ED trends over 5 years pre- and post community service changes

\begin{tabular}{|c|c|c|c|c|c|c|}
\hline Period & & $\begin{array}{l}2013-2014 \text { before } \\
\text { change }\end{array}$ & $\begin{array}{l}2014-2015 \text { after } \\
\text { change }\end{array}$ & $\begin{array}{l}2015- \\
2016\end{array}$ & $\begin{array}{l}2016- \\
2017\end{array}$ & $\begin{array}{l}2017- \\
2018\end{array}$ \\
\hline Number seen & & 544 & 1118 & 1075 & 1049 & 1062 \\
\hline \multirow[t]{3}{*}{ Mode of referral $n(\%)$} & Self-referral & $419(77 \%)$ & $664(59 \%)$ & $690(64 \%)$ & $667(64 \%)$ & $701(66 \%)$ \\
\hline & GP referral & $86(16 \%)$ & $363(33 \%)$ & $308(29 \%)$ & $289(28 \%)$ & $301(28 \%)$ \\
\hline & Other & $39(7 \%)$ & $91(8 \%)$ & $77(7 \%)$ & $93(8 \%)$ & $60(6 \%)$ \\
\hline Number without physical issues (\%) & & $159(29 \%)$ & $582(52 \%)$ & $563(52 \%)$ & $547(52 \%)$ & $539(51 \%)$ \\
\hline Length of stay hours (SD) & & $14.9(8.6)$ & $11.1(8.1)$ & $11.6(8.8)$ & $10.1(7.4)$ & $10.4(6.4)$ \\
\hline Did not wait & & $11(2 \%)$ & $26(2.3 \%)$ & $16(1.5 \%)$ & $8(0.8 \%)$ & $17(1.6 \%)$ \\
\hline
\end{tabular}


processed though the longer ED care pathway, when their needs could be more appropriately met in a different setting. Overcrowding in the ED is a worsening problem, which impacts negatively on patient care. Numerous studies show that ED overcrowding is associated with poorer patient outcomes including higher mortality rates [10-12, 17]. ED overcrowding is also associated with medication delays and errors [18, 19] with one study finding that medication errors in psychiatric patients in the ED was as high as 65\% [20]. Overcrowding is also associated with increased burnout in healthcare professionals [21], and the psychological wellbeing of clinicians in Ireland, as in many countries, is already at critical point [22]. This has consequences for recruitment and retention-already a concerning issue in the medical workforce [23, 24].

Perhaps what is most striking in this study - in the year after the service change, the proportion of patients presenting with no physical issue identified at triage increased proportionally from 29.2 to $52.1 \%$, and remained high over subsequent years. This is a worrying trend - the ED is typically a stimulating and stressful environment - not a therapeutic milieu for distressed individuals - and in some cases, can precipitate iatrogenic harm $[25,26]$. Staff numbers may be insufficient to provide the intensive nursing input or care support that may be required in high risk cases, and ED clinicians often lack appropriate confidence and training in such scenarios [26]. Importantly, patients have also outlined their preference not to be seen in ED when presenting in crisis [27]. The policy on this issue is clear. The NCP for Patients presenting to ED following Self- Harm recommends that patients without physical health problems (for example suicidal ideation without self-harm) should have direct access to psychiatric assessment in the community without recourse to ED [5]. Additionally, the 2012 National Emergency Medicine Programme report states clearly that the ED must "not be the pathway of access to mental health care for patients with mental ill-health who have no acute medical need" [4]. Both programmes advise that primary care be the first point of contact for such patients, and further recommend implementation of a formalised system for primary care to access emergency mental health services in such cases.

Our study showed that the average length of stay (LOS) in ED reduced by $3.8 \mathrm{~h}$ from 14.9 to 11.1 , and the proportion of patients who did not wait to be seen reduced slightly, though the latter was not statistically significant. These findings are likely to reflect the beneficial impact of implementation of the NCP for self-harm [5], which led to the appointment of extra self-harm nurses, in addition to the Emergency Medicine Programme for Ireland, which set out a target that $95 \%$ of patients should be either admitted or discharged within 6-h of arriving at an ED [4]. Despite the modest improvements in ED LOS shown in our study, an average time spent in ED of over $10 \mathrm{~h}$ is far from ideal, much of which will include long waiting time to be seen. We did not have data on the waiting times of the offsite psychiatric unit to directly compare; however, it is likely that waiting times were much shorter here given the lack of additional steps of triage and ED physician assessment prior to specialist mental health review. While it must be acknowledged that many patients with medical conditions have lengthy waiting times, emergencies such as stroke, seizures, and myocardial infarction have typically little to no waiting time, and psychiatric emergencies can also be life threatening $[28,29]$. In the UK, it has been shown that people presenting with a mental health issue are over six times more likely than people presenting with a physical concern to wait more than $4 \mathrm{~h}$ at the ED [30]. Other studies have highlighted that patients awaiting psychiatric admission typically undergo a significantly prolonged ED LOS compared with other patient groups, which entails a considerable significant financial impact on services [31,32]. This is an unacceptably poorer standard of care for patients with mental health needs. Like all health conditions, prompt access to appropriate service is critical for patient outcomes, and crucially, it has been reported that timely access to psychiatric assessment may reduce the risk of subsequent self-harm and suicide [33]. Longer ED waiting times have also been associated with increased risk of violence towards staff [34].

Our study showed that proportionally, the number of GP referrals increased significantly compared with a decreased proportion of self-referrals after the service change. This is an interesting finding and challenges a possible assumption held by some that patients may self-refer excessively for mental health treatment, and raises the possibility that GPs may find it difficult to access pathways to urgent mental health assessments, alternative to the ED. Bari et al. [35] surveyed the views of GPs around crisis mental health assessmentfinding the majority of GPs reported difficulty accessing community mental health teams (CMHTs) during working hours, and it was been observed that this stems from a resourcedriven inability to provide an emergency service in the community setting [36]. The importance of optimising primary care services has been highlighted for this group [37], with studies suggesting that up to $80 \%$ of patients who completed suicide had contact with primary in the 12 months prior to suicide versus $31 \%$ who had been in contact with mental health services [38]. Such findings emphasise the importance of embedding the relevant interventions that address self-harm and suicide prevention within the primary care setting. This is difficult to sufficiently implement with an inadequately resourced system - the Irish 2020 mental health budget comprises a mere $6 \%$ of the overall health budget compared with a $13 \%$ average across the European Union $[39,40]$.

Overall, there have been limited studies describing the impact of the relocation of community psychiatric services on emergency departments, and there is a gap in research on the clinical effectiveness and cost-effectiveness of crisis care in mental health [41]. Crisis intervention and home treatment teams have not shown convincing evidence for improving 
outcomes for either mental health ED visits or psychiatric inpatient admissions [42-44]. A study by Parwani et al. [15] showed ED LOS and psychiatric admission rates decreased when patients were seen directly by a multidisciplinary team in a psychiatric decision unit (PDU), in cases where the primary complaint was psychiatric without concomitant medical issues. In Australia, the introduction of a specialised behavioural assessment unit (BAU) facilitated more timely and direct access to a mental health clinician, reduced use of restrictive interventions and decreased ED LOS [14]. A large multicentre study is ongoing at present in the UK to evaluate the impact of PDUs, with a particular focus on ED LOS and inpatient psychiatric admission outcomes [45]. To the authors' knowledge, such units are not available in Ireland.

\section{Strengths and limitations}

To the authors' knowledge at the time of writing, there is little evidence in the existing literature examining the impact of a community mental health service relocation on the ED, and following this trend over time. The numbers in our study are high, and the findings are of particular relevance given the constant overcrowding crisis in the EDs with the associated negative repercussions for patients and staff, compounded further by the recent pandemic. This study is a single site study that took place in a large tertiary centre, however is likely to be relevant to other similar general hospital settings. The clinical information on the data set only comprised triage notes and did not record the exact nature of the medical problem, psychiatric diagnoses, or other factors, however this was consistent with the purpose of the study which was to evaluate the patients requiring ED physical evaluation. We did not have data from the stand-alone unit to compare, however the purpose was to evaluate the relocation impact from the perspective of the ED service.

\section{Conclusion}

Parity of esteem in care for mental health patients remains a concerning and neglected issue. Whilst some patients presenting with psychiatric emergencies will require an ED physician assessment, the majority are best assessed and managed outside the stressed ED environments, within primary care and community mental health care settings. This study highlights the need for the development of appropriate pathways of care both within and separate from the ED for this patient group. Such developments cannot be implemented without the appropriate resourcing of community-based services, which often struggle to provide appropriate, evidence-based care for patients with severe and enduring mental disorders on a sparsely allocated budget. This is now a matter of critical urgency given we are in a post Covid world, where infection control and crowding are of utmost importance, and the ways in which we physically provide healthcare will have stark ramifications for our patients and healthcare workers.

Acknowledgments The authors would like to sincerely thank Lorraine Carey, Management Information Officer, Beaumont Hospital.

Data availability Data will be made available to interested individuals upon formal request made to authors.

\section{Compliance with ethical standards}

Conflict of interest The authors declare that they have no conflict of interest.

Ethical disclosure statement This was a service evaluation study. No contact was made with patients for the duration of the study. No identifiable patient data was used. Data was obtained via an electronic hospital system and anonymised to ensure patient confidentiality. Data was stored on a fire-walled computer with coded access.

\section{References}

1. APA Task Force on Psychiatric Emergency Services (2002) Report and recommendations regarding psychiatric emergency and crisis services: a review and model program descriptions. American Psychiatric Association, Arlington

2. Lofchy J, Boyles P, Delwo J (2015) Emergency psychiatry: clinical and training approaches. Can J Psychiatry 60(7):1-7

3. National Collaborating Centre for Mental Health (2004) (UK N. Self-harm: the short-term physical and psychological management and secondary prevention of self-harm in primary and secondary care. Leicester, UK: The British Psychological Society \& The Royal College of Psychiatrists

4. Clinical Strategy and Programmes Directorate, Health Service Executive (2012) The National Emergency Medicine Programme: a strategy to improve safety, quality, access and value in Emergency Medicine in Ireland. Royal College of Surgeons in Ireland, Dublin, p 188

5. Health Service Executive (2016) National clinical programme for the assessment and management of patients presenting to emergency departments following self-harm. The National Clinical Programme for Mental Health Clinical Programme, HSE Clinical Strategy and Programmes Division

6. Gibbons P, Lee A, Parkes J, Meaney E (2012) Value for money: a comparison of cost and quality in two models of adult mental health service provision. Health Service Executive, Dublin

7. Zun L (2016) Care of psychiatric patients: the challenge to emergency physicians. Western J Emergency Med 17(2):173-176

8. Anderson E, Nordstrom K, Wilson M, Peltzer-Jones J, Zun L, Ng A, Allen M (2017) American Association for Emergency Psychiatry Task Force on medical clearance of adults part I: introduction, review and evidence-based guidelines. Western J Emergency Med 18(2):235-242

9. Wilson M, Nordstrom K, Anderson E, Ng A, Zun L, Peltzer-Jones J, Allen M (2017) American Association for Emergency Psychiatry Task Force on medical clearance of adult psychiatric patients. Part II: controversies over medical assessment, and consensus recommendations. Western J Emergency Med 18(4):640-646

10. Sprivulis P, Da Silva J, Jacobs I, Frazer A, Jelinek G (2006) The association between hospital overcrowding and mortality among 
patients admitted via Western Australian emergency departments. Med J Aust 184(12):616-616

11. Richardson D (2006) Increase in patient mortality at 10 days associated with emergency department overcrowding. Med J Aust 184(5):213-216

12. Guttmann A, Schull M, Vermeulen M, Stukel T (2011) Association between waiting times and short-term mortality and hospital admission after departure from emergency department: population-based cohort study from Ontario, Canada. BMJ 342(jun01 1):d2983

13. Nesper A, Morris B, Scher L, Holmes J (2016) Effect of decreasing county mental health services on the emergency department. Ann Emerg Med 67(4):525-530

14. Braitberg G, Gerdtz M, Harding S, Pincus S, Thompson M, Knott J (2017) Behavioural assessment unit improves outcomes for patients with complex psychosocial needs. Emergency Med Australas 30(3):353-358

15. Parwani V, Tinloy B, Ulrich A, D'Onofrio G, Goldenberg M, Rothenberg C, Patel A, Venkatesh AK (2018) Opening of psychiatric observation unit eases boarding crisis. Acad Emerg Med 25(4):456-460

16. Mental Health Commission. Inspectorate of mental health services catchment team report. Mental Health Commission; 2013 p. 1.available at https://www.mhcirl.ie/File/IRs/car2013 nb.pdf

17. Plunkett P, Byrne D, Breslin T, Bennett K, Silke B (2011) Increasing wait times predict increasing mortality for emergency medical admissions. Eur J Emerg Med 18(4):192-196

18. Kulstad E, Sikka R, Sweis R, Kelley K, Rzechula K (2010) ED overcrowding is associated with an increased frequency of medication errors. Am J Emerg Med 28(3):304-309

19. Mitchell Scott B, Considine J, Botti M (2014) Medication errors in ED: do patient characteristics and the environment influence the nature and frequency of medication errors? Australas Emerg Nurs J 17(4):167-175

20. Bakhsh H, Perona S, Shields W, Salek S, Sanders A, Patanwala A (2014) Medication errors in psychiatric patients boarded in the emergency department. Int J Risk Saf Med 26(4):191-198

21. Boyle A, Beniuk K, Higginson I, Atkinson P (2012) Emergency department crowding: time for interventions and policy evaluations. Emergency Med Int 2012:1-8

22. Hayes B, Prihodova L, Walsh G, Doyle F, Doherty S (2019) Doctors don't do-little: a national cross-sectional study of workplace well-being of hospital doctors in Ireland. BMJ Open 9(3): e025433

23. Brugha, RF.; Cronin, F; Clarke, N; Group, RCSI health workforce research: retaining our doctors medical workforce evidence, 201318. Challenges and Responses. Royal College of Surgeons in Ireland. Report 2018. https://doi.org/10.25419/rcsi.10776611.v1

24. Pidd S (2003) Recruiting and retaining psychiatrists. Adv Psychiatr Treat 9(6):405-411

25. Bender D, Pande N, Ludwig M. https://aspe.hhs.gov/basic-report/ literature-review-psychiatric-boarding\#note81. 2008 [cited 4 May 2020]. Available from: http://aspe.hhs.gov/basic-report/ literature-review-psychiatric-boarding

26. Stefan S (2006) Emergency department treatment of the psychiatric patient. Oxford, New York

27. Thomas K, Owino H, Ansari S, Adams L, Cyr J, Gaynes B et al (2018) Patient-centered values and experiences with emergency department and mental health crisis care. Adm Policy Ment Health Ment Health Serv Res 45(4):611-622

28. Mavrogiorgou P, Brüne M, Juckel G (2011) The management of psychiatric emergencies. Deutsches Aerzteblatt Online

29. Tueth M, Zuberi P (1999) Life-threatening psychiatric emergencies in the elderly: overview. J Geriatr Psychiatry Neurol 12(2):60-66
30. Care Quality Commission (2014) Key findings for the national accident and emergency patient survey 2014. Care Quality Commission, London

31. Nicks B, Manthey D (2012) The impact of psychiatric patient boarding in emergency departments. Emergency Med Int 2012:1-5

32. Talbott J (2012) Trends in the duration of emergency department visits, 2001-2006. Yearbook of Psychiatry and Applied Mental Health 2012, pp 203-204

33. Hickey L, Hawton K, Fagg J, Weitzel H (2001) Deliberate selfharm patients who leave the accident and emergency department without a psychiatric assessment: a neglected population at risk of suicide. J Psychosom Res 50(2):87-93

34. Morphet J, Griffiths D, Plummer V, Innes K, Fairhall R, Beattie J (2014) At the crossroads of violence and aggression in the emergency department: perspectives of Australian emergency nurses. Aust Health Rev 38(2):194-201

35. Bari QA., Carey C, Russell V, Barry H, Wilkinson C, Browne L, Kelly S, MacHale S 2019. If you build it they will come, Beaumont hospital emergency department as a mental health referral option for general practitioners. Joint Conference of the College of Psychiatrists of Ireland and the Royal College of Psychiatrists of Northern Ireland Winter Conference 2019, Belfast, Northern Ireland (Unpublished work)

36. Health Service Executive (2017) Review of the Operation of the Programme 2017: National Clinical Programme for the Assessment and Management of Patients Presenting to the Emergency Department following Self-Harm. Health Service Executive, Mental Health Division

37. Parsonage M, Fossey M, Tutty C (2012) Liaison psychiatry in the modern NHS. Centre for Mental Health, London

38. Stene-Larsen K, Reneflot A (2017) Contact with primary and mental health care prior to suicide: a systematic review of the literature from 2000 to 2017. Scand J Public Health 47(1):9-17

39. Cullen K, McDaid D, Wynne R, Matosevic T and Park A-L (2017) A wide-angle international review of evidence and developments in mental health policy and practice. Evidence review to inform the parameters for a refresh of A Vision for Change (AVFC). Retrieved from http://health.gov.ie/

40. Mental Health Reform Pre-Budget Submission 2020. Retrieved June 162020 from https://www.mentalhealthreform.ie/prebudget-2020/

41. Paton F, Wright K, Ayre N, Dare C, Johnson S, Lloyd-Evans B, Simpson A, Webber M, Meader N (2016) Improving outcomes for people in mental health crisis: a rapid synthesis of the evidence for available models of care. Health Technol Assess 20(3):1-162

42. Jacobs R, Barrenho E (2011) Impact of crisis resolution and home treatment teams on psychiatric admissions in England. Br J Psychiatry 199(1):71-76

43. Jespersen S, Lawman B, Reed F, Hawke K, Plummer V, Gaskin C (2016) The impact of integrating crisis teams into community mental health services on emergency department and inpatient demand. Psychiatry Q 87(4):703-712

44. Wheeler C, Lloyd-Evans B, Churchard A, Fitzgerald C, Fullarton $\mathrm{K}$, Mosse L et al. (2015) Implementation of the crisis resolution team model in adult mental health settings: a systematic review. BMC Psychiatry 15(1)

45. Goldsmith L, Smith J, Clarke G, Anderson K, Lomani J, Turner K et al (2020) What is the impact of psychiatric decision units on mental health crisis care pathways? Protocol for an interrupted time series analysis with a synthetic control study. BMC Psychiatry 20(1)

Publisher's note Springer Nature remains neutral with regard to jurisdictional claims in published maps and institutional affiliations. 